\title{
Effects on Depression and Anxiety After Mid-Urethral Sling Surgery for Female Stress Urinary Incontinence
}

This article was published in the following Dove Press journal: Research and Reports in Urology

\section{Manami Kinjo \\ Kazuki Masuda \\ Yu Nakamura \\ Satoru Taguchi (1D \\ Mitsuhiro Tambo (D) \\ Takatsugu Okegawa \\ Hiroshi Fukuhara}

Department of Urology, Kyorin University School of Medicine, Tokyo I8I-86II, Japan
Correspondence: Manami Kinjo

Tel +8I $4224755 \mathrm{II}$

Fax +8I 422428431

Email kinjo_I702@yahoo.co.jp
Purpose: Urinary incontinence (UI) is a common health-related problem in the female population and has a negative impact on many aspects of patients' quality of life including mental problems. This study evaluated the mental effects of mid-urethral sling (MUS) surgery in female patients with stress UI or stress UI-dominant mixed UI.

Patients and Methods: Women with stress UI or stress UI-dominant mixed UI who underwent MUS surgery and were enrolled in this study and followed up for 12 months. The International Consultation of Incontinence Questionnaire-Short Form (ICIQ-SF) and the Hospital Anxiety and Depression Scale (HADS) at baseline and 12 months after the MUS to evaluate the efficacy of the surgery.

Results: At baseline, proportions of the patients with anxiety (HADS-Anxiety score of $\geq 8$ ) and depression (HADS-Depression score of $\geq 8)$ were $21.6 \%(22 / 102)$ and $24.5 \%(25 / 102)$, respectively. At 12 months postoperatively, the median ICIQ-SF score, HADS-Anxiety score, and HADS-Depression score were significantly improved compared with their baseline values $(p<0.001$ for ICIQ-SF and HADS-Depression, $p=0.011$ for HADS-Anxiety). At 12 months postoperatively, changes in the ICIQ-SF and HADS-A scores showed a moderate correlation $(r=0.578, p<0.001)$, and changes in the ICIQ-SF and HADS-D scores had a strong correlation $(\mathrm{r}=0.838, \mathrm{p}<0.001)$.

Conclusion: This study demonstrated that MUS surgery significantly improved UI, anxiety, and depression with significant correlations in their symptoms. This suggests that improvement of the patients' UI helped to relieve their symptoms of anxiety and depression.

Keywords: urinary incontinence, anxiety, depression, mid-urethral sling surgery

\section{Introduction}

Urinary incontinence (UI) is the complaint of involuntary leakage of urine ${ }^{1}$ and is a common health problem in the female population. ${ }^{2-4}$ Stress UI (SUI) is reportedly the most common type of UI in female population, but urgency UI (UUI) and mixed UI (MUI) are also frequently encountered. Generally, the prevalence of UI increases with age, SUI is more common in young and middle-aged women and UUI and MUI predominate in older women. ${ }^{5}$ UI has a negative impact on many aspects of patients' quality of life, including their economic status, daily activities, personal relationships, and mental health. ${ }^{6,7}$

Depression and/or anxiety have an estimated worldwide prevalence of $10 \%$ and cause not only human health problems but also economic burdens. ${ }^{8,9}$ Many largescale studies have demonstrated a relationship between depression and/or anxiety 
and UI. ${ }^{6,7,10-12}$ Women with UI are more likely to have depression and/or anxiety than women without UI. ${ }^{6,7,11}$ And higher severity in UI was associated higher mental condition. ${ }^{13}$ With respect to the type of incontinence, studies have shown that the rate of depression is higher in patients with UUI and MUI than in patients with SUI. ${ }^{11,12}$ Moreover, some research has shown that treatment of UI improves not only UI symptoms but also the mental status, especially in patients with UUI that is treated pharmacologically. ${ }^{14-16}$ However, few reports have provided details about the influence of SUI surgery on the mental status. Additionally, whether surgical treatment for SUI will improve the mental status, such as anxiety and/or depression, remains unclear especially in the long term.

This study was performed to evaluate the efficacy of mid-urethral sling (MUS) surgery not only for UI symptom but also its effects on anxiety and depression symptoms evaluated by specific questionnaire in patients with SUI or SUI-dominant MUI.

\section{Materials and Methods}

This study was conducted in accordance with the Good Clinical Practice guidelines and the principles of the Declaration of Helsinki after being approved by the Kyorin University ethics committee (approval H27-135). Written informed consent was obtained from all individual participants included in the study. This prospective observational study was performed from June 1, 2015 to March 31, 2018. Study involved women with SUI or SUI-dominant MUI aged $\geq 20$ years who underwent MUS surgery (both retropubic and transobturator) in our institution and were followed up for 12 months. SUI and SUI-dominant MUI patients selected MUS surgery after conservative treatment (behavioral therapy and/or pharmacotherapy) that was not effective and after expressing a desire to have surgery. The choice of either the retropubic or transobturator approach was at each patient's discretion' after they had received an explanation of each of the procedure. However, if a patient had a history of abdominal open surgery, we selected the transobturator approach. The preoperative evaluation included assessment of the patients' characteristics, medical history, physical examination findings (including a transvaginal examination), cough stress test with at least $250 \mathrm{~mL}$ of saline in the bladder, 3-day bladder diary, uroflowmetry, and post-void residual urine (PVR) volume. SUI was confirmed in all patients by a positive cough stress test, the International Consultation of Incontinence
Questionnaire-Short Form (ICIQ-SF) ${ }^{17}$ and at least one episode of SUI recorded in a 3-day bladder diary. SUI dominant MUI was confirmed by ICIQ-SF score, fewer UUI episodes than SUI episodes recorded in the 3-day bladder diary, and more patient-reported distress in SUI symptoms. The exclusion criteria were a planned future pregnancy, having neurogenic disease, any concomitant surgery (such as prolapse repair), a surgical history of UI, PVR volume of $>100 \mathrm{~mL}$, and psychiatric diagnosis and medical treatment of depression or other psychiatric disorder.

The objective outcome was measured using transvaginal examination (the presence or absence of mesh exposure or pain), cough stress test, uroflowmetry, and PVR volume 12 months after the surgery. The subjective outcome was measured using the following questionnaires before and 12 months after the surgery: The ICIQ-SF, and the Hospital Anxiety and Depression Scale (HADS).$^{18}$ The HADS is a validated tool for detecting anxiety and depression in non-psychiatric patients and a score of $\geq 8$ is consistent with clinical anxiety/depression. The HADS has been validated in Japanese language. ${ }^{19}$

Cure was defined as the objective outcome of a negative stress test result, and the subjective outcome of no self-reported SUI symptoms.

\section{Statistical Analysis}

A sample size calculation was performed before beginning the study. One study showed that after SUI surgery, the mean HADS-Anxiety (HADS-A) and HADS-Depression (HADSD) scores changed from 5.0 to 2.6 and from 4.1 to 2.3 , respectively. ${ }^{20}$ At $95 \%$ statistical power and a significance level of 0.05 , we determined that the sample size should be 47 patients for evaluation of the HADS-A score and 88 patents for evaluation of the HADS-D score. At an estimated dropout rate of 20\%, 100 patients needed to be enrolled.

The Wilcoxon signed-rank test was used to compare differences in the ICIQ-SF and HADS scores, and the McNemar test was used to compare the prevalence of anxiety/depression at baseline and at 12 months. Spearman's rank correlation coefficient was used to identify correlations between ICIQ-SF and HADS scores. Statistical analyses were conducted with SAS software (SAS Institute, Cary, NC, USA) and a $p$ value of $<0.05$ was considered statistically significant.

\section{Results}

In total, 114 patients met the inclusion criteria, and of these, $102(89.5 \%)$ patients were analyzed in the study. We 
excluded 10 patients due to incomplete questionnaire responses and 2 patients for lost of follow up; thus, 12 patients were excluded from the analysis. Two patients were prescribed antidepressants for insomnia; however, they were not diagnosed with depression/anxiety nor did they consult with psychiatrist, therefore, they were included in the analysis. The patients' demographic and baseline characteristics are shown in Table 1. The patients' median age, median body mass index, and median parity were 61 years (range, $38-88$ years), $23 \mathrm{~kg} / \mathrm{m}^{2}$, (range, 16.8-34.9), and 2 children (range, 0-3), respectively. Seventy-five patients $(73.5 \%)$ were postmenopausal. Fifty-nine patients (57.8\%) were diagnosed with SUI and 43 (42.2\%) were diagnosed with SUI-dominant MUI. The median baseline ICIQ-SF score was 13 (range, 4-21). The median baseline HADS-A score was 4 (range, $0-15$ ), and 21.6\% (22/102) of patients were diagnosed with clinical anxiety (HADS-A score of $\geq 8$ ). The median HADS-D score was 5 (range, $1-14)$, and $24.5 \%(25 / 102)$ of patients were diagnosed with clinical depression (HADS-D score of $\geq 8$ ). Before the surgery, the ICIQ scores showed a weak positive correlation with HADS-A scores $(r=0.308, p=0.030)$ and a moderate positive correlation with HADS-D scores $(\mathrm{r}=0.558, \mathrm{p}<$ $0.001)$.

Table 2 shows the change in symptoms from baseline to 12 months. At 12 months postoperatively, the patients' median ICIQ-SF total score, median HADS-A score, and median HADS-D score were significantly improved compared with their baseline values (ICIQ-SF: 13.0 to $0.0, \mathrm{p}<0.001$; HADS-A: 4.0 to $3.0, \mathrm{p}=0.011$; HADS-D: 5.0 to $3.0, \mathrm{p}<$ $0.001)$. The rate of diagnosis of clinical anxiety significantly decreased from $21.6 \%$ to $10.2 \%(p=0.042)$, and the rate of

Table I Patients' Characteristics

\begin{tabular}{|l|l|}
\hline Patients, $\mathbf{n}$ & 102 \\
\hline Age, years & $61(33-88)$ \\
BMI, $\mathrm{kg} / \mathrm{m}^{2}$ & $23.0(16.8-34.9)$ \\
Parity & $2(0-3)$ \\
Postmenopausal & $75(73.5 \%)$ \\
ICIQ-SF total score & $13(4-21)$ \\
HADS-A score & $4(0-15)$ \\
HADS-D score & $5(0-14)$ \\
HADS-A score of $\geq 8$ & $22(21.6 \%)$ \\
HADS-D score of $\geq 8$ & $25(24.5 \%)$ \\
\hline
\end{tabular}

Note: Data are presented as medians (range) or $\mathrm{n}(\%)$.

Abbreviations: BMI, body mass index; ICIQ-SF, International Consultation of Incontinence Questionnaire-Short Form; HADS-A, Hospital Anxiety and Depression Scale-Anxiety; HADS-D, Hospital Anxiety and Depression ScaleDepression.
Table 2 Changes in Symptom Scores from Baseline

\begin{tabular}{|l|l|l|l|l|}
\hline & Baseline & \multicolumn{2}{l|}{12 Months } \\
\cline { 2 - 5 } & Score & Score & $\begin{array}{l}\text { Change from } \\
\text { Baseline }\end{array}$ & p value \\
\hline ICIQ-SF & $13.0(4$ to 21$)$ & $0(0$ to 16$)$ & $-9(-21$ to 3$)$ & $<0.001$ \\
HADS-A & $4.0(0$ to 15$)$ & $3.0(0$ to 13$)$ & $-1.0(-8$ to 6$)$ & 0.011 \\
HADS-D & $5.0($ (I to 14) & $3.0(0$ to 15) & $-2.0(-10$ to 14) & $<0.001$ \\
\hline
\end{tabular}

Note: Data are presented as medians (range). $\mathrm{p}$ values were obtained with the Wilcoxon signed-rank test.

Abbreviations: ICIQ-SF, International Consultation of Incontinence Questionnaire-Short Form; HADS-A, Hospital Anxiety and Depression ScaleAnxiety; HADS-D, Hospital Anxiety and Depression Scale-Depression.

clinical depression also significantly decreased from $24.7 \%$ to $8.2 \%(\mathrm{p}<0.001)$. Regarding urodynamic parameters, both the median voided volume and the median PVR volume showed no change from before to 12 months after the MUS surgery (voided volume: 252.1 to $257.4 \mathrm{~mL}, \mathrm{p}=$ 0.362; PVR volume: 10 to $15 \mathrm{~mL}, \mathrm{p}=0.052$ ). However, the median maximum flow rate significantly decreased (30.1 to $20.2 \mathrm{~mL} / \mathrm{s}, \mathrm{p}<0.001)$.

The results of the correlation analysis of changes in the ICIQ-SF and HADS scores that accompanied surgery are shown in Table 3. At 12 months, changes in the ICIQ-SF and HADS-A scores showed a moderate correlation $(\mathrm{r}=0.578$, $p<0.001$ ), and the changes in the ICIQ-SF and HADS-D scores showed a strong correlation $(\mathrm{r}=0.838, \mathrm{p}<0.001)$.

Before the surgery, 21.6\% (22/102) and 24.5\% (25/ 102) of patients were clinically diagnosed with anxiety and depression, respectively. At 12 months after the MUS surgery, $59 \%(13 / 22)$ and $80 \%(20 / 25)$ of patients no longer had anxiety or depression. However, $10.8 \%$ (11/ $102)$ and $8.8 \%(9 / 102)$ of the total population had anxiety and depression 12 months after the surgery. Like the UI symptoms, the rates of anxiety and depression were significantly lower compared with before the MUS surgery (anxiety: $21.6 \%$ to $10.8 \%, p=0.003$; depression: $24.5 \%$ to $8.8 \%, \mathrm{p}<0.001)$. Of 11 patients who had anxiety at 12 months after the surgery, $81.8 \% \quad(9 / 11)$ remained

Table 3 Correlation of Changes in ICIQ-SF and HADS Scores

\begin{tabular}{|l|l|l|}
\hline & \multicolumn{2}{|l|}{12 Months } \\
\cline { 2 - 3 } & Correlation Coefficient & p value \\
\hline Variation of HADS-A score & 0.578 & $<0.001$ \\
Variation of HADS-D score & 0.838 & $<0.001$ \\
\hline
\end{tabular}

Abbreviations: ICIQ-SF, International Consultation of Incontinence Questionnaire-Short Form; HADS-A, Hospital Anxiety and Depression ScaleAnxiety; HADS-D, Hospital Anxiety and Depression Scale-Depression. 
unchanged from baseline, and 18.2\% (2/11) newly developed anxiety symptoms. Indeed, of nine patients with depression at 12 months after the surgery, 55.5\% (5/9) remained unchanged from baseline, and 44.4\% (4/9) newly developed depression symptoms.

During the 12-month postoperative observation period, the complications were, de novo overactive bladder symptoms in seven patients $(6.9 \%)$ and persistent mild groin pain for more than 1 month in one patient $(1.0 \%)$ who had treated with transobturator approach (no treatment required, discharged at 3 months). Two patients complained of weak urine stream, but neither intermittent catheterization nor medication was required, because the PVR volume was $<100 \mathrm{~mL}$. No mesh tape exposure or dyspareunia was observed. Twelve patients $(11.6 \%)$ reported persistence of SUI at 12 months with no treatment required. However, two patients required tape shortening under local anesthesia at 4 and 10 months, respectively, which completely resolve the UI.

\section{Discussion}

In the present study, MUS surgery significantly improved UI and both anxiety and depression symptoms in patients with SUI or SUI-dominant MUI. Indeed, the changes in the UI symptoms were moderately correlated with anxiety and strongly correlated with depression. This suggests that improvement of UI symptoms helps to relieve anxiety and especially depression symptoms. To the best of our knowledge, this is the first study to evaluate the effects of MUS surgery on UI, and both anxiety and depression in Japanese women with SUI or SUI-dominant MUI for a relatively long term.

Preoperatively, $20.6 \%$ and $24.5 \%$ of participants had anxiety and depression symptoms, respectively, despite our exclusion of patients with clinically diagnosed depression or other psychiatric disorders. The lifetime prevalence rates of anxiety and depression in Japan have been estimated as $8.1 \%$ and $6.1 \%$, respectively. ${ }^{21}$ Despite the fact that the definitions of anxiety and depression are not exactly the same, patients who are planning to undergo SUI surgery have an anxiety rate more than 2.5 times higher and a depression rate approximately 4 times higher than the general Japanese population.

Two large epidemiological surveys in Norway $^{10}$ and three in Western countries (Sweden, United Kingdom, and United States) ${ }^{22}$ reported a high prevalence of anxiety and depression in participants with UI diagnosed by the HADS using the same definition. The prevalence of anxiety according to the type of UI was $22.9 \%$ to $30.4 \%$ in patients with SUI, $28.1 \%$ to $30.2 \%$ in patients with UUI, and $32.0 \%$ to $49.1 \%$ in patients with MUI. The prevalence of depression according to the type of UI was $9.0 \%$ to $16.8 \%$ in patients with SUI, $11.7 \%$ to $17.8 \%$ in patients with UUI, and $16.9 \%$ to $34.7 \%$ in patients with MUI. Indeed, among 373 participants with UI attending the eHealth program, which aims to improve healthcare using information and communication technology, the prevalence of anxiety was $12.4 \%$ in patients with SUI and $13.8 \%$ in those with UUI/MUI, and the prevalence of depression was $3.2 \%$ in patients with SUI and $10.6 \%$ in those with UUI/MUI evaluated using the same definition. $^{22}$ Differences in the prevalence of anxiety/ depression may have been affected by UI severity or racial or cultural factors. For example, the average ICIQ score in participants in the eHealth program was 10.4 in those with SUI and 11.6 in those with UUI/MUI; however, the median ICIQ score was 13.0 in our study population, indicating more severe UI symptoms. Patients planning to undergo surgery for UI may have more severe UI symptoms and a considerably higher prevalence of anxiety/ depression.

Some studies have focused on surgical treatment for SUI and its effect on mental health. Rosenzweig et $\mathrm{al}^{23}$ reported that successful surgical treatment significantly improved sleep disturbance and tension, whereas unsuccessful results significantly worsened depression and sleep disturbance in 63 patients with SUI. Stoffel et $\mathrm{al}^{24}$ also reported that UI symptoms after SUI surgery were a risk factor for depressive symptoms. In the present study, persistent SUI symptoms were present in 11patients (nine with SUI only, two with MUI) 12 months after surgery without any intervention; three patients experienced relief from depression, one experienced relief from anxiety, respectively; two experienced persistent anxiety, and one was newly diagnosed with anxiety. Among seven patients with de novo UUI, two experienced relief from anxiety and depression, respectively; one experienced persistence of both anxiety and depression; and no cases of new onset anxiety/depression were observed. In our comparison of 80 patients with no UI and 22 patients with any kind of UI (nine with SUI, seven with UUI, two with MUI, and six with other types of UI) at 12 months after surgery, the prevalence rate of anxiety was $11.3 \%$ (9 of 80 patients) among patients with no UI and $9.1 \%$ (2 of 22 patients) among patients with any type of UI, with no significant difference $(p=0.378)$. Furthermore, the depression rate 
was $8.9 \%$ (7 of 80 patients) among patients with no UI and $9.1 \%$ ( 2 of 22 patients) among patients with any type of UI, with no significant difference between the two groups $(p=0.428)$ (data not shown). The presence of any type of UI after SUI surgery did not worsen the prevalence of a poor mental health status in our study. This suggests that there is no need for intervention in patients with persistent SUI (even after MUS surgery), that the SUI symptoms are mild, that patients are satisfied with their treatment, and that their UI symptoms do not adversely affect their mental status. Indeed, the median ICIQ-SF score changed significantly and meaningfully (a 5-point change in ICIQ-SF score is considered the minimum clinically important difference) from 13.5 (range, 5-21) to 5.0 (range, 3-11) $(\mathrm{p}<0.001)$ after surgery in patients with persistent UI. If precise and repeated explanations are provided of the UI pathophysiology (especially of differences between SUI and UUI) the possibility of persistence of some types of UI, and the methods of resolving these conditions, then patients can tolerate their condition and remain satisfied with their outcomes. Balzarro et al demonstrated that good preoperative counseling facilitates better patient's management and reducing patients disaffection. ${ }^{25}$ In addition, it is also possible that persistent mental health problems are affected not only UI symptoms, but also by another factors (eg, genetic, personal, or environmental factors). In another study of the positive effect of surgical treatment for SUI on patients' mental status, Black et $\mathrm{al}^{26}$ found that $63 \%$ of patients had mental health-related problems before the treatment and that this proportion significantly decreased to $39 \%$ at 12 months after several types of surgical procedures for SUI. The present study also demonstrated that the prevalence of anxiety decreased by almost half $(21.6 \%$ to $10.8 \%)$ and that the prevalence of depression decreased by about onethird $(24.5 \%$ to $8.8 \%)$. Despite the different prevalence of mental health conditions arising from differences in definitions, surgical procedures, and racial and cultural backgrounds, both studies indicate that surgical treatment for SUI has a positive effect on patients' mental health. Furthermore, we evaluated not only the changes in the rates and scores, but also the correlation between UI symptoms and mental health symptoms. In this sample of women with SUI or SUI-dominant MUI, a change in UI symptoms was moderately correlated with a change in anxiety symptoms $(r=0.578, p<0.001)$ and strongly correlated with a change in depression symptoms $(r=$ $0.838, \mathrm{p}<0.001)$. Thus, we suggest that UI symptoms relief also improves anxiety/depression. Siff et $\mathrm{al}^{27}$ demonstrated the effect of major depression on the outcome of MUS surgery in a secondary analysis of the TOMUS trial, a randomized equivalence trial in which 597 women with SUI were assigned to either retropubic or transobturator MUS surgeries. These authors hypothesized that baseline major depression would negatively affect improvement in quality of life and UI severity after MUS surgery. However, MUS surgery improved UI symptoms equally and improved incontinence-specific quality of life significantly more in patients with than in those without major depression. Both their findings and our own suggest that if mental health symptoms are affected by UI symptoms, amelioration in UI could also improve mental health. A large 10-year longitudinal population-based survey in Norway demonstrated that UI at baseline was significantly associated with the incidence of both anxiety and depression, ${ }^{10}$ supporting our hypothesis that UI affects mental health symptoms. Similar to the present study, another study evaluating the efficacy of surgical treatment for SUI on both depression and anxiety. ${ }^{20}$ That study concluded that surgical treatment improved quality of life and anxiety but not depression. Differences between those findings and our own may stem from differences in study design, such as the short study period ( 8 weeks) and small sample (32 patients). Recovery from depression may require more time; 8 weeks may have been too short to evaluate the depression, resulting in the lack of a significant change in depression symptoms. Involuntary loss of urine causes patients to avoid social events for fear of urine loss in public, and they therefore experience a feelings of isolation. ${ }^{28}$ Additionally, patients with UI often feel embarrassment and, negative selfesteem because of social stigma. All these factors may lead to the development of anxiety and depression.

This study had several limitations. First, we have no control group, however, the moderate to strong correlations in score changes between UI and depression/anxiety suggested a close relationship between UI and patients' mental health. The small sample and single-center study design are additional limitations. However, despite these limitations, we used validated questionnaires to assess UI and mental health status, a prospective study design, and a relatively long follow-up period. MUS surgery for patients with SUI or SUI-dominant MUI may improve both UI symptoms and psychiatric symptoms, which is positive news for SUI patients and their physicians. A longer follow up may clarify the relationship between 
UI and mental health after MUS for SUI and SUIdominant MUI patients.

\section{Conclusion}

This study demonstrated that MUS surgery significantly improved UI and anxiety/depression symptoms. Improvements in UI symptoms were correlated with improvements in anxiety and especially depression. This information may be useful for both patients and physicians in planning SUI surgery.

\section{Acknowledgments}

The authors thank all study participants and medical staff for their assistance in data collection. The authors also thank Angela Morben, DVM, ELS, and Diane Williams, PhD, from Edanz Group, and Dr. Motofumi Suzuki for editing a draft of this manuscript. This paper was presented at the 49th ICS Annual Meeting as a poster presentation talk with interim findings.

\section{Disclosure}

The authors report no conflicts of interest in this work.

\section{References}

1. Haylen BT, de Ridder D, Freeman RM, et al. An International Urogynecological Association (IUGA)/International Continence Society (ICS) joint report on the terminology for female pelvic floor dysfunction. Neurourol Urodyn. 2010;29:4-20. doi:10.1002/nau.20798

2. Irwin DE, Milsom I, Hunskaar S, et al. Population-based survey of urinary incontinence, overactive bladder, and other lower urinary tract symptoms in five countries: results of the EPIC study. Eur Urol. 2006;50:1306-1314. doi:10.1016/j.eururo.2006.09.019

3. Homma Y, Yamaguchi O, Hayashi K. Neurogenic bladder society committee. Epidemiologic survey of lower urinary tract symptoms in Japan. Urology. 2006;68:560-564. doi:10.1016/j.urology.2006.03.035

4. Coyne KS, Sexton CC, Thompson CL, et al. The prevalence of lower urinary tract symptoms (LUTS) in the USA, the UK and Sweden: results from the Epidemiology of LUTS (EpiLUTS) study. BJU Int. 2009;104:352-360. doi:10.1111/j.1464-410X.2009.08427.x

5. Hannestad YS, Rortveit G, Sandvik H. Norwegian EPINCONT study. A community-based epidemiological survey of female urinary incontinence: the Norwegian EPINCONT study. Epidemiology of Incontinence in the County of Nord-Trøndelag. $J$ Clin Epidemiol. 2000;53:1150-1157. doi:10.1016/S0895-4356(00)00232-8

6. Melville JL, Walker E, Katon W, et al. Prevalence of comorbid psychiatric illness and its impact on symptom perception, quality of life, and functional status in women with urinary incontinence. $\mathrm{Am}$ J Obstet Gynecol. 2002;187:80-87. doi:10.1067/mob.2002.124839

7. Coyne KS, Kvasz M, Ireland AM, et al. Urinary incontinence and its relationship to mental health and health-related quality of life in men and women in Sweden, the United Kingdom, and the United States. Eur Urol. 2012;61:88-95. doi:10.1016/j.eururo.2011.07.049.

8. Baxter AJ, Scott KM, Vos T, et al. Global prevalence of anxiety disorders: a systematic review and meta-regression. Psychol Med. 2013;43:897-910. doi:10.1017/S003329171200147X
9. Ferrari AJ, Somerville AJ, Baxter AJ, et al. Global variation in the prevalence and incidence of major depressive disorder: a systematic review of the epidemiological literature. Psychol Med. 2013;43: 471-481. doi:10.1017/S0033291712001511

10. Felde G, Ebbesen MH, Hunskaar S. Anxiety and depression associated with urinary incontinence. A 10-year follow-up study from the Norwegian HUNT study (EPINCONT). Neurourol Urodyn. 2017;36 (2):322-328. doi:10.1002/nau.22921

11. Felde G, Bjelland I, Hunskaar S. Anxiety and depression associated with incontinence in middle-aged women: a large Norwegian cross-sectional study. Int Urogynecol J. 2012;23:299-306. doi:10.1007/s00192-011-1564-3

12. Hansson Vikström N, Wasteson E, Lindam A, et al. Anxiety and depression in women with urinary incontinence using E-health. Int Urogynecol J. 2020;24. doi:10.1007/s00192-020-04227-2.

13. Siddiqui NY, Wiseman JB, Cella D, et al. Mental health, sleep and physical function in treatment seeking women with urinary incontinence. J Urol. 2018;200:848-855. doi:10.1016/j.juro.2018. 04.076

14. Kinjo M, Yamaguchi T, Tambo M, et al. Effects of mirabegron on anxiety and depression in female patients with overactive bladder. Urol Int. 2019;102:331-335. doi:10.1159/000497282

15. Rogers R, Bachmann G, Jumadilova Z, et al. Efficacy of tolterodine on overactive bladder symptoms and sexual and emotional quality of life in sexually active women. Int Urogynecol J Pelvic Floor Dysfunct. 2008;19:1551-1557. doi:10.1007/s00192-008-0688-6

16. Kafri R, Kodesh A, Shames J, et al. Depressive symptoms and treatment of women with urgency urinary incontinence. Int Urogynecol J. 2013;24:1953-1959. doi:10.1007/s00192-0132116-9

17. Gotoh M, Homma Y, Funahashi Y, et al. Psychometric validation of the Japanese version of the International consultation on incontinence questionnaire-short form. Int J Urol. 2009;16:303-306. doi:10.1111/ j.1442-2042.2008.02237.x

18. Zigmond AS, Snaith RP. The hospital anxiety and depression scale. Acta Psychiatr Scand. 1983;67:361-370. doi:10.1111/j.1600-0447.19 83.tb09716.x

19. Hatta H, Higashi A, Yoshiro H, et al. A validation of hospital anxiety and depression scale. Jpn J Psychosom Med. 1998;38:309-315.

20. Innerkofler PC, Guenther V, Rehder P, et al. Improvement of quality of life, anxiety and depression after surgery in patients with stress urinary incontinence: results of a longitudinal short-term follow-up. Health Qual Life Outcomes. 2008;29(6):72. doi:10.1186/1477-75256-72.

21. Ishikawa H, Kawakami N, Kessler RC, et al. World Mental Health Japan Survey Collaborators. Lifetime and 12-month prevalence, severity and unmet need for treatment of common mental disorders in Japan: results from the final dataset of World Mental Health Japan Survey. Epidemiol Psychiatr Sci. 2016;25:217-229. doi:10.1017/ S2045796015000566

22. Coyne KS, Kvasz M, Ireland AM, et al. Urinary incontinence and its relationship to mental health and health-related quality of life in men and women in Sweden, the United Kingdom, and the United States. Eur Urol. 2012;61:88-95. doi:10.1016/j.eururo.2011.07.049

23. Rosenzweig BA, Hischke D, Thomas S, et al. Stress incontinence in women. Psychological status before and after treatment. $J$ Reprod Med. 1991;36:835-838.

24. Stoffel JT, Morgan D, Dunn R, et al. Urinary incontinence after stress incontinence surgery: a risk factor for depression. Urology. 2009;73:41-46. doi:10.1016/j.urology.2008.08.478

25. Balzarro M, Rubilotta E, Goss C, et al. Counseling in urogynecology: a difficult task, or simply good surgeon-patient communication? Int Urogynecol J. 2018;29:943-948. doi:10.1007/s00192-018-3673-8

26. Black N, Griffiths J, Pope C, et al. Impact of surgery for stress incontinence on morbidity: cohort study. BMJ. 1997;6(315): 1493-1498. doi:10.1136/bmj.315.7121.1493 
27. Siff LN, Jelovsek JE, Barber MD. The effect of major depression on quality of life after surgery for stress urinary incontinence: a secondary analysis of the Trial of Midurethral Slings. Am J Obstet Gynecol. 2016;215:455.e1-9. doi:10.1016/j.ajog.2016.04. 039
28. Molinuevo B, Batista-Miranda JE. Under the tip of the iceberg: psychological factors in incontinence. Neurourol Urodyn. 2012; 31:669-671. doi:10.1002/nau.21216

\section{Publish your work in this journal}

Research and Reports in Urology is an international, peer-reviewed, open access journal publishing original research, reports, editorials, reviews and commentaries on all aspects of adult and pediatric urology in the clinic and laboratory including the following topics: Pathology, pathophysiology of urological disease; Investigation and treatment of urological disease; Pharmacology of drugs used for the treatment of urological disease. The manuscript management system is completely online and includes a very quick and fair peer-review system, which is all easy to use. Visit http://www.dovepress.com/ testimonials.php to read real quotes from published authors. 